\title{
CVD diamond growth mechanisms as identified by surface topography
}

\author{
W. J. P. van Enckevort, G. Janssen, W. Vollenberg, J. J. Schermer and L. J. Giling \\ Experimental Solid State Physics III, University of Nijmegen, Toernooiveld, NL-6525 ED Nijmegen ( Netherlands) \\ M. Seal \\ Sigillum B.V., Guido Gezellestraat 5. NL-1077 WN Amsterdam (Netherlands)
}

\begin{abstract}
The surface morphologies of numerous homoepitaxial, chemical-vapour-deposition-grown diamond films have been examined by phase-sensitive optical microscopy. The layers were produced by the hot filament technique as well as by the acetylene-oxygen combustion method. The $\{111\}$ and $\{100\}$ faces manifest themselves as $F$ faces below the roughening temperature and grow via steps nucleated at three-dimensional diamond particles or at dislocations. The rate-determining step in $1111 ;$ diamond growth is a hindered surface diffusion of the growth species towards the steps. The layer-by-layer growth on the 100 ; faces is discussed in terms of $(2 \times 1)$ surface reconstruction in combination with the presence of a 4, screw axis. The $\{113$ face on flame-grown diamonds is made up of strong $\langle 110\rangle$ chains of bonds which are interconnected by weak forces due to surface reconstruction. The slight curvature of this face points to an $\mathrm{F}$ face close to the roughening point. The 1110$\}$ face is rough, i.e. K S type, and no layer growth occurs. The different modes of crystal growth as well as local differences in step spacing are replicated as variations in the intensity of band $\mathrm{A}$ and $575 \mathrm{~nm}$ cathodoluminescence.
\end{abstract}

\section{Introduction: mechanisms of crystal growth}

From the theory of crystal growth it is well known [1] that crystal surfaces can be classified into two categories: (1) rough faces with a zero step free energy; (2) flat faces with a positive step free energy.

The growth of the rough crystal faces proceeds via a more or less random addition of growth units at the surface. This means that there is no correlation between adjacent surface areas and the surface shows no tendency to flatten. Since no energy barrier exists for the formation of new growth layers, the rough faces grow fast.

The growth of flat crystal surfaces proceeds layerwise, involving lower and higher steps. Owing to the finite step free energy, an activation barrier has to be overcome to form a new growth layer. Therefore flat faces are the slowest-growing ones and determine the final crystal shape. The new growth layers are generated by twodimensional nucleation, by contact nucleation at foreign particles or by spiral growth. The successive steps in the growth of an $\mathrm{F}$ face are mass transport, surface diffusion and integration of growth units at the steps. Which step determines the velocity of growth differs from case to case.

According to the periodic bond chain (PBC) theory [2], the flat faces are characterized by two or more interconnected chains of strong bonds (PBCs) within a layer thickness $d_{h k l}(h k l$ corrected for the systematic extinctions of the space group in question [2]). These so-called $F$ faces are in fact webs of interwoven PBCs. The step energy is finite. Monte Carlo simulations and theoretical analysis [3] have shown that for lower intermolecular (atomic) energies $\varphi$, for high coverages with impurities and for higher temperatures $T$ the step free energy vanishes and a flat surface turns rough. The critical ratio $(T / \varphi)_{c}$ at which the transition occurs is called the roughening point. The other faces with one or no PBC within $d_{\mathrm{hk} 1}$ are denoted as $\mathrm{S}$ and $\mathrm{K}$ faces respectively. These faces are rough.

For the diamond structure the only $\mathrm{PBC}$ is the zigzag $\backslash \mathrm{C} / \mathrm{C} \backslash \mathrm{C} /$ chain parallel to $\langle 110\rangle$. From this it follows [4] that the only $F$ face is $\{111\}$, the forms in the zones $\langle 110\rangle$ (excluding $\{111 ;$ and $\{100\})$ are $\mathrm{S}$ type and the remaining faces (including $\{100\}$ ) are $K$ type. Since the morphology of a crystal is determined by the slowestgrowing faces, the theoretical form of diamond is octahedral, i.e. bounded by the $\{111\} \mathbf{F}$ faces.

In some cases $\mathrm{K}$ and $\mathrm{S}$ faces appear as flat, stepped surfaces. This is caused by the creation of extra surface PBCs due to the adsorption of impurities or by surface reconstruction [5]. Here a positive edge free energy is generated and growth proceeds layer by layer.

\section{Diamond growth and characterization}

The homoepitaxial layers used for surface examination were deposited by the hot-filament-assisted chemical 
vapour deposition (HF-CVD) technique and by the oxygen-acetylene combustion (flame) method [6]. Single-crystal natural type IIa diamonds were used as substrates. For the HF-CVD growth experiments $\{100\}$ and $\{110\}$ plates $3-5 \mathrm{~mm}$ in diameter and $0.25 \mathrm{~mm}$ thick were cut and polished smooth to within $3^{\circ}$ of the exact orientation. The $\{111\}$ plates were cleaved and were, apart from the cleavage steps, exact in orientation. The substrates used for flame growth were cylinders $1-5 \mathrm{~mm}$ wide and $0.25 \mathrm{~mm}$ thick, which were soldered on top of a molybdenum substrate holder. The top and bottom faces of the cylinders were $\{110\}$ or $\{100\}$.

The experimental set-up for $\mathrm{HF}$ growth has been detailed elsewhere [7-9]. The crystals were grown under a wide range of conditions. The substrate temperature, as measured by IR pyrometry after correction for the influence of the filament, was varied between 735 and $915^{\circ} \mathrm{C}$. The error in absolute temperature might be as large as $50^{\circ} \mathrm{C}$, but the relative errors are within $10{ }^{\circ} \mathrm{C}$. The $\mathrm{CH}_{4}: \mathrm{H}_{2}$ volume ratio was varied between $0.67 \%$ and $5 \%$, keeping the total pressure fixed at 50 mbar and the flow rate at 5 standard $\mathrm{cm}^{3} \mathrm{~s}^{-1}$. In standard runs a WC filament heated at $1910 \pm 10^{\circ} \mathrm{C}$ was used, but higher deposition rates $\left(2-7 \mu \mathrm{m} \mathrm{h}^{-1}\right)$ were obtained by taking a TaC filament at $T=2325 \pm 10^{\circ} \mathrm{C}$.

The substrate holder in the HF reactor was made of hexagonal boron nitride. This is a source of contamination in the gas phase which leads to boron doping of the diamond films [9]. As shown by Wang et al. [10], the addition of boron compounds in a microwaveplasma-assisted CVD system changes the surface morphology and the defect structure of the growing crystallites. Although during the present experiments no clear relation between surface structure and boron content was found, it cannot be excluded that the observed morphologies differ from undoped material.

The arrangement for flame growth is almost identical to that described in refs. 9 and 11. To improve the substrate temperature control, the molybdenum substrate holder was cooled by periodic jets of water [12]. By changing the duty cycle of the water supply, a temperature stability to within $10^{\circ} \mathrm{C}$ was obtained. The diamond temperature as measured by two-colour IR pyrometry was varied between about 600 and $1120^{\circ} \mathrm{C}$, with an absolute error of $20^{\circ} \mathrm{C}$ and a reproducibility of $10^{\circ} \mathrm{C}$. The acetylene supersaturation $\left(S_{\mathrm{ac}}\right)$ ranged from $2.0 \%$ to $6.0 \%$. Here $S_{\text {ac }}$ is defined as the acetylene flow minus the acetylene flow of an ideal welding flame, divided by the latter. The ideal welding flame, for which the acetylene feather has just disappeared, was found by visual inspection.

The surface structures of the grown films were observed by Nomarski differential interference-contrast microscopy (NICM) and phase-shifting interferometry (PSI). By these techniques of phase-sensitive optical microscopy height differences down to about $1 \mathrm{~nm}$ can be imaged [13, 14]. The distribution of luminescent point defects was investigated by placing the specimens in a chamber for cathodoluminescence $(\mathrm{CL})$ examination by low and medium magnification optical microscopy [15]. The position-dependent CL spectra were recorded via the microscope which was coupled to a monochromator-photomultiplier unit by an optical fibre.

\section{Flat faces}

During the present investigations of the HF- and flame-grown diamond specimens only two orientations yielded flat faces with a positive edge free energy, namely $\{111\}$ and $\{100\}$. Both forms show a strong tendency to flatten and grow layerwise via steps. To verify all other possible orientations, additional deposition runs were carried out using hemispherical [8] and cylindrical [16] single-crystal diamond substrates. No additional flat faces were found.

\section{1. $\{111\}$ faces}

For all deposition runs the HF-CVD-grown $\{111\}$ faces are covered by rounded, shallow growth hillocks (Fig. 1). The inclination of the side walls is about $0.7^{\circ}$. The presence of the hillocks points to a layer mechanism for the growth of $\{111\}$ diamond, as predicted by the PBC theory. The individual growth steps are too low or too closely spaced to be resolved by SEM and NICM, but have recently been revealed by scanning tunnelling microscopy (STM) [17] and reflection electron microscopy (REM) [18]. Since in nearly all cases no foreign particles occur at the growth centres, it can safely be concluded that the steps are generated by lattice defects rather than by contact nucleation.

In addition to growth hillocks, one can also see

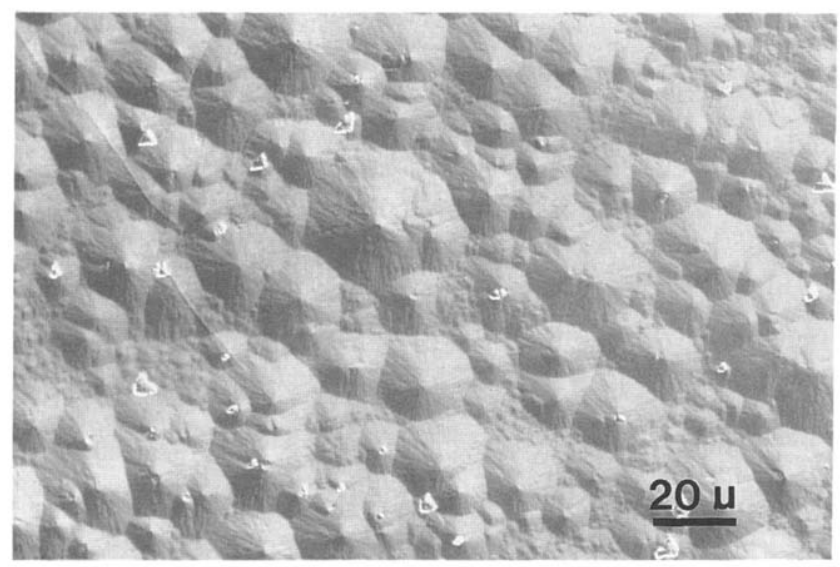

Fig. 1. Characteristic pattern of shallow, rounded growth hillocks on a diamond film deposited on a $\{111\}$ substrate (NICM). The layer was grown by HF-CVD $\left(T_{\text {sub }} \approx 800^{\circ} \mathrm{C}, T_{\text {fil }}=1910^{\circ} \mathrm{C}, \mathrm{CH}_{4}: \mathrm{H}_{2}=0.64 \%\right)$. 
overgrown cleavage steps on the $\{111\}$ faces. It is interesting that, regardless of substrate temperature, the tops of all the macrosteps show a sharp discontinuity in slope (Fig. 2). As elaborated elsewhere [19] in terms of the kinematic wave theory of step propagation [20], this only occurs if the velocity of steps decreases for increasing step densities. This points to transport-limited growth. Rau and Picht [21] showed that gas phase transport determines CVD growth in a low pressure microwave reactor. In the present HF system, however, growth is limited by surface processes instead of volume diffusion, because (1) the deposition rate of layers on $\{110\}$ substrates is two to five times higher than on $\{100\}$ and $\{111\}$ plates [9] and (2) despite the fact that rough faces (which have no tendency to flatten) grow faster in areas of increased supersaturation, no enhanced growth was observed near the edges of the substrate even for the thickest $\mathrm{S} / \mathrm{K}$-type layers $\{110\}$. Therefore the ratelimiting step in the growth of $\{111\}$ diamond by HFCVD must be the surface diffusion of growth units towards the steps. In view of the high surface coverage with atomic $\mathrm{H}$ and the large adsorption energy of $\mathrm{CH}_{n}$, diffusion will indeed be very difficult.

Since the surface diffusion coefficient $[D]$ is a symmetric two-dimensional tensor and the point group of the top layer on 111 diamond is $3 \mathrm{~m} 1$, then according to Neumann's rule, $[D]$ must be isotropic [14]. Therefore, if surface diffusion is rate limiting, the propagation rate of a step on 1111$\}$ diamond is independent of lateral orientation. This agrees with the observed rounded form of the growth hillocks.

For flame-grown 1111 surfaces grown at elevated substrate temperatures the growth hillocks are triangular (Fig. 3). This non-rounded form indicates that step integration now plays an essential role in determining the growth velocity. The hillocks are bounded by [112] steps. As has been elaborated by Okada et al. [22], this

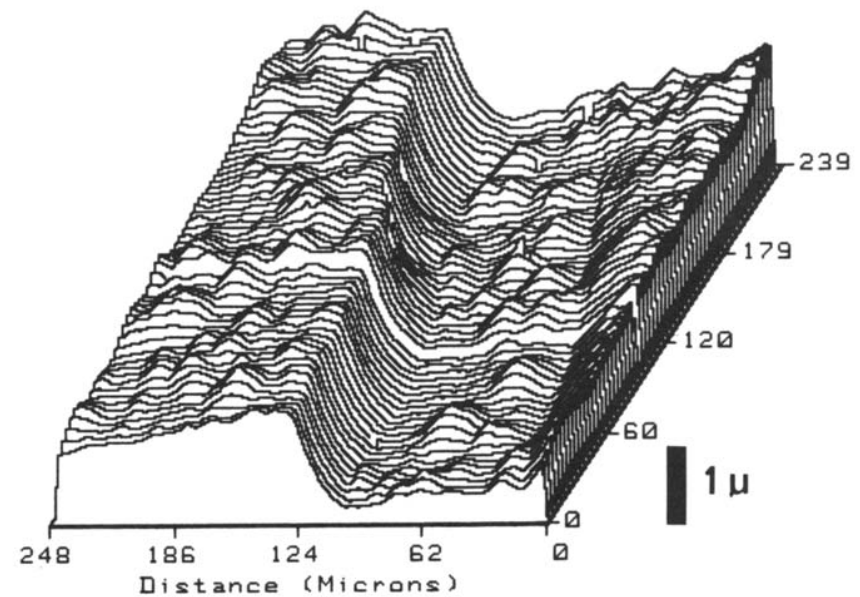

Fig. 2. PSI topograph showing the profile of a step bunch developed from a cleavage step on a $\{11\}$ diamond surface. In addition. numerous shallow growth hillocks are imaged (same specimen as in Fig. 1).

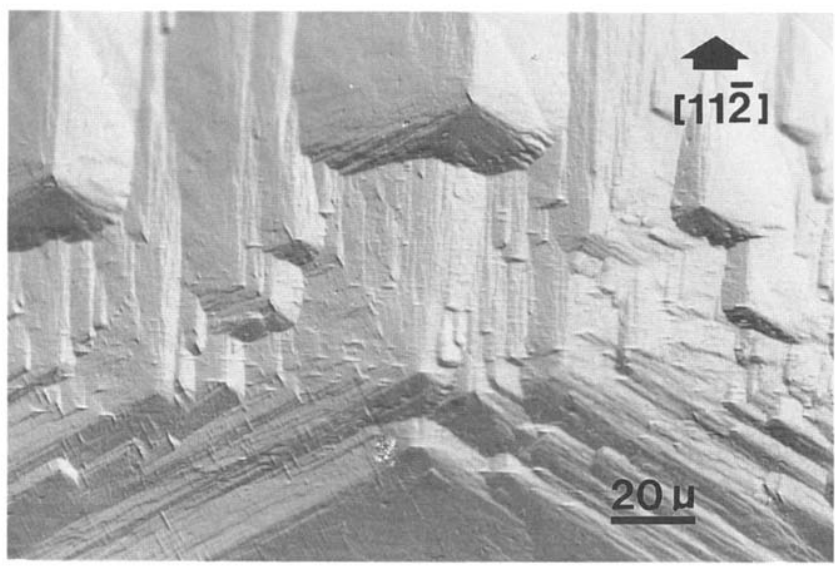

Fig. 3. Triangular hillocks bounded by [112] steps on the 1111 face of a diamond single crystal grown by the flame method $\left(T_{\text {diamond }}=\right.$ $\left.1120^{\circ} \mathrm{C}, S_{\mathrm{ac}}=4.0 \%\right)$. The micrograph was taken by NICM

points to the occurrence of step reconstruction involving dimer formation [5].

After prolonged growth (greater than $5 \mu \mathrm{m}$ ) the $\{111$ : faces of both the HF- and flame-grown crystals become multiply twinned. Since the steps cannot pass the twin boundaries, this constrains the growth to small islands $[19,23]$. Finally (above $40 \mu \mathrm{m}$ ), the films become polycrystalline [9].

\section{2. $\{100 ;$ faces}

Similarly to the films on 1111$\}$, the layers grown on $\{100\}$ substrates are also covered by numerous shallow hillocks (Figs. 4 and 5). For both HF-CVD and flame growth the elevations are square with edges parallel to the PBC directions $\langle 110\rangle$. This indicates a step mechanism for $\{100\}$ diamond growth. The steps are nucleated at foreign particles $[7,24]$ or originate from lattice defects. The latter was confirmed by the direct observation of growth spirals $[19,25]$.

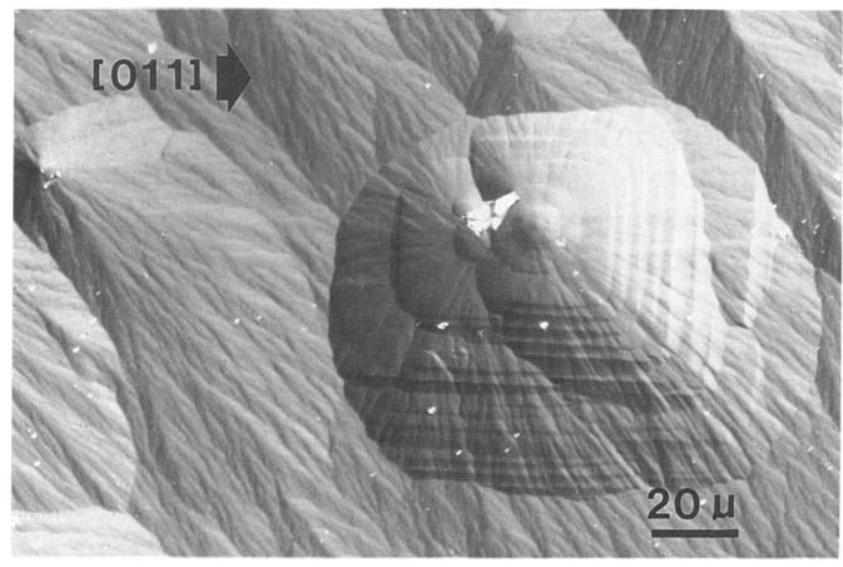

Fig. 4. NICM photograph showing growth hillocks on a thicker $(20 \mu \mathrm{m}): 100$ film produced by HF-CVD. 


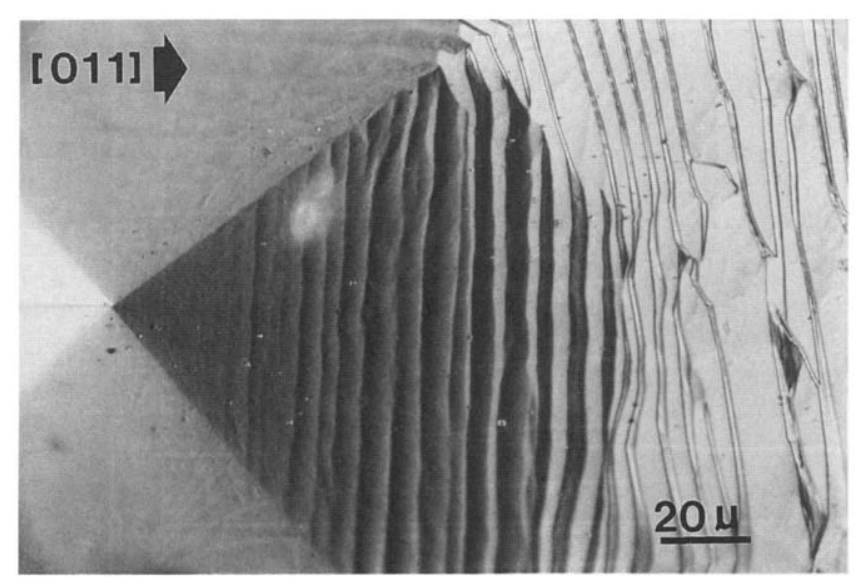

Fig. 5. Development of a macrostep pattern on the $\{100\}$ face of a flame-grown diamond $\left(T_{\text {diamond }}=1200^{\circ} \mathrm{C}, S_{\mathrm{ac}}=4.0 \%\right)$. The micrograph was taken by NICM.

The growth velocity of $\{100\}$ diamond is determined by the most active step sources. This is a struggle for life in which the smaller hillocks are overwhelmed by the step trains from the dominating ones. Many of the overgrown centres can be recognized as the feather-like patterns in Fig. 4.

For flame-grown layers step bunching, i.e. a pile-up of lower steps into higher ones, often plays an essential role. From the appearance of the smooth side faces near the summit of the hillock shown in Fig. 5, one can infer that just after emission the steps are low and evenly spaced. After prolonged propagation, i.e. further away from the growth centre, bunching occurs and finally macrosteps develop. Such a coarsening of steps during crystallization has been discussed by van der Eerden and Müller-Krumbhaar [26] and is explained by assuming that the velocity of a step depends on the time that a terrace in front of the step has been exposed to the ambient phase containing a certain amount of impurities.

For the HF-CVD-grown $\{100\}$ layers it was shown that the advancement rate of steps is independent of step spacing [19]. This means that the integration of growth units or surface diffusion over a distance far less than the separation between adjacent steps $(2-4 \mathrm{~nm})$ is rate limiting in $\{100\}$ diamond growth.

According to the PBC theory, $\{100\}$ diamond is a $\mathrm{K}$ face and should be rough. This is in conflict with the observed surface structures. However, as shown by STM $[23,27]$, the $\{100\}$ surface is not a simple truncated bulk phase but exhibits a $(2 \times 1)$ surface reconstruction. Owing to the creation of extra surface bonds, this lowers the surface free energy and creates a positive edge free energy: the rough face turns flat $[5,24]$. As follows from direct observation $[23,27]$, the thinnest layer on a $(2 \times 1)$-reconstructed $\{100\}$ diamond or silicon surface is $d_{400}$. The two-dimensional point group symmetry of such a slice is $\mathrm{mm}$ and no fourfold axis occurs. This implies that the advancement velocity of a [110] step in such a layer differs from the perpendicular step [110]. STM of Si [28] and diamond [23] shows that steps parallel to the dimer rows (type A steps [29]) are straight and advance slower than the perpendicular B steps which are serrated. If steps are emitted from a growth centre, the fast type B steps will catch up the slower type A steps ahead and double-height steps are formed (Fig. 6). Double-height growth layers have been observed on $\{100\}$ silicon surfaces grown by molecular beam epitaxy [27]. To fulfil the $4_{1}$ symmetry, unit steps must be present between each set of adjacent, perpendicular double-height ledges. This effect is known as "step interlacing" [30]. The above shows that although each individual layer lacks a fourfold axis, the overall form of a growth hillock must be fourfold symmetric. This agrees with the observations.

\section{Rough faces}

Apart from $\{111\}$ and $\{100\}$, all the other faces on CVD-grown diamonds are rough and show no strong tendency to flatten. Two faces have been examined in detail, namely $\{113\}$ and $\{110\}$.

\section{1. $\{113\}$ faces}

On flame-grown diamond layers deposited on cylindrical $\{110\}$ substrates at $T<1200^{\circ} \mathrm{C}$ a curved band appears on the rim of the cylinder between the planar $\{111\}$ and $\{100\}$ faces. At higher temperatures and prolonged growth this band develops into a $\{113\}$ facet [16]. As can be inferred from Fig. 7, the $\{113\}$ forms are covered by steps parallel to the PBC directions $\langle 1 \overline{1} 0\rangle$. The faces are planar along $\langle 1 \overline{1} 0\rangle$ but are slightly curved in the perpendicular direction $\langle 33 \overline{2}\rangle$.

Following the first-bond-order PBC theory, the $\{113\}$ face is $\mathrm{S}$ type. In addition, the polar plot of broken bond

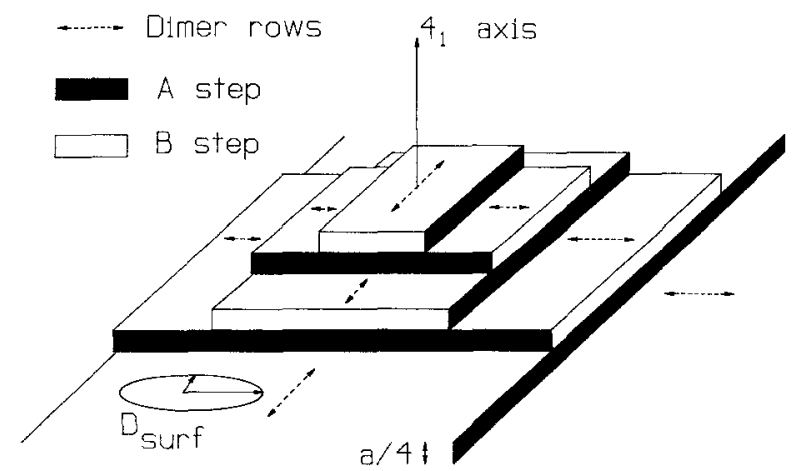

Fig. 6. The formation of a growth hillock on $\{100\}$ diamond with double-height steps, $2 d_{400}$, as a result of the simultaneous occurrence of $(2 \times 1)$ surface reconstruction and a $4_{1}$ axis perpendicular to the growth face. 


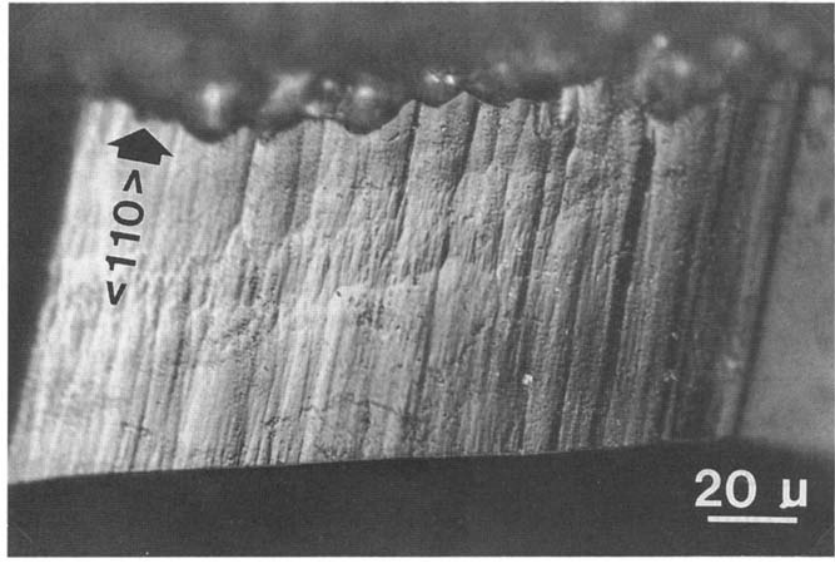

Fig. 7. NICM photograph of a $\{113\}$ face of a flame-grown diamond. The crystal was grown in $1 \mathrm{~h}$ at $1190 \mathrm{C}$ and $S_{\mathrm{ac}}=6.0 \%$.

energy $v$ s. orientation in the $\langle 110\rangle$ zone shows no local minimum at $\{113\}[31]$. This means that this face should show no tendency to flatten. Therefore the observed stabilization of $\{113\}$ can only be explained by the occurrence of weak surface bonds which interconnect the $\langle 110\rangle$ PBCs. The extra surface bonds are probably introduced by surface reconstruction in a comparable way as has been proposed for the isostructural $\{113\}$ silicon surface [31]. Recently, reconstruction of $\{113 ; \mathrm{Si}$ has indeed been observed by STM [32].

Since the $\{113\}$ faces on diamond are slightly curved and developed from a curved band rather than being formed instantaneously on the cylinder, they should be considered as rough, i.e. as $F$ faces near the roughening transition. For increasing deposition temperatures the $\{113\}$ faces become more and more planar. This is explained by the fact that at the elevated temperatures the surface coverage decreases and surface reconstruction, which is necessary for the creation of the extra surface bonds, becomes more important.

\subsection{0$\}$ faces}

The diamond layers deposited on the $\{110\}$ substrates are invariably rough. Neither a tendency to flatten nor evidence for layer growth, e.g. shallow hillocks or steps, was found. The surface morphology of the $\{110$ f films is mainly determined by layer thickness [19]. For the thinnest films (approximately $5 \mu \mathrm{m}$ or less) a granular structure can be seen by SEM and NICM [7-9]. STM [17] and atomic force microscopy (AFM) [33] show an extended hill and valley structure which indicates the onset of microfaceting. As shown in Fig. 8, layers of intermediate thickness $(5-15 \mu \mathrm{m})$ exhibit a clear pattern of microfacets. Finally, flame-grown layers exceeding $25 \mu \mathrm{m}$ in thickness are characterized by a coarse pattern of hills and facets $10-100 \mu \mathrm{m}$ wide and $20-40 \mu \mathrm{m}$ high.

The presence of a hill and valley pattern is common for an overgrown $\mathrm{S}$ or $\mathrm{K}$ face. For example, microfacets

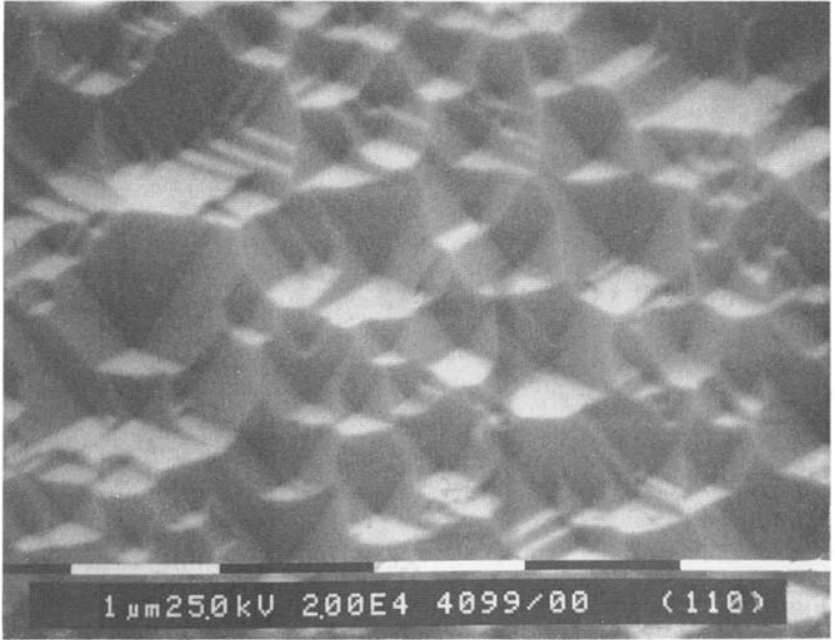

Fig. 8. Pattern of microfacets on a diamond film deposited on a 110 ; substrate (SEM). The layer was grown hy HF-CVD $\left\{T_{\text {sub }}=825 \mathrm{C}\right.$, $\left.T_{\text {Ii }}=2325 \mathrm{C}, \mathrm{CH}_{4}: \mathrm{H}_{2}=1.5 \%\right)$.

are also formed on $1001 ; \mathrm{KH}_{2} \mathrm{PO}_{4}$ seeds immersed in at supersaturated aqueous solution [34]. From PBC analysis it is known that this face is $\mathrm{K} / \mathrm{S}$ type [35]. The observed roughening on a scale of $100 \mathrm{~nm}$ and upwards is explained by the high surface free energy of an $\mathrm{S}$ or $\mathrm{K}$ face. To lower its energy, such an atomically rough face breaks up into facets of lower surface free energy. Despite an increase in surface area. a net gain in surface free energy is obtained.

\section{Cathodoluminescence topography}

Since a substrate holder machined from hexagonal boron nitride was used during the HF-CVD growth experiments, a certain amount of boron was incorporated into the grown layers [9]. This leads to green band A CL caused by electron-hole recombination at donor-boron pairs [36]. is shown elsewhere [8], the intensity of this luminescence varies strongly with substrate orientation. Therefore it was concluded that the incorporation of impurities affecting green band A CL is largely determined by the surface processes during crystallization.

A relation between green band A luminescence and crystal growth was also found on a microscopic scale. The CL topograph in Fig. 9 shows a pattern of shallow growth hillocks on a slightly misoriented $\{100\}$ substrate. The intensity of luminescence is maximal for the hillocks, medium for the misoriented surface and minimal for the semiplanar summits of some hillocks. In other words, a decrease in step spacing leads to an increase in CL intensity. A possible cause is the time-dependent adsorption of luminescence quenching centres between the steps. If the separation is large, more impurities are 


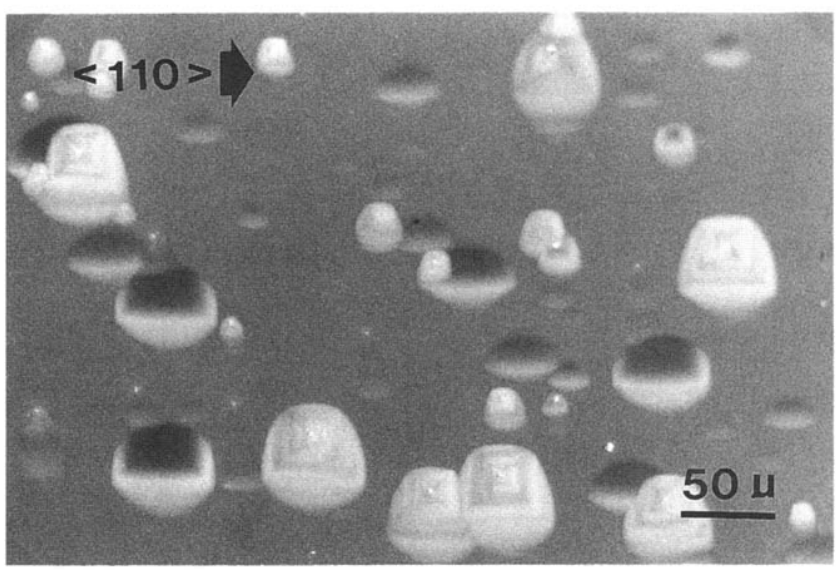

Fig. 9. CL topograph of an HF-CVD-grown specimen $\left(T_{\text {sub }}=825^{\circ} \mathrm{C}\right.$, $T_{\mathrm{fil}}=2325^{\circ} \mathrm{C}, \mathrm{CH}_{4}: \mathrm{H}_{2}=3 \%$ ) showing an increase in luminescence intensity for decreasing step distances on $\{100\}$.

For flame-grown diamond the CL is dominated by the orange-red $575 \mathrm{~nm}$ system which has been attributed to nitrogen-vacancy pairs [37] as well as by the blue band A emission from donor-acceptor pairs [38]. To gather information on the $\mathrm{CL}$ as a function of the direction of diamond growth, an epitaxial layer was deposited on a hemispherical, monocrystalline diamond substrate. A CL image of the flame-grown hemisphere is given in Fig. 10. The $\{111\}$ and $\{100\}$ faces as well as the $\langle 110\rangle$ zones between these faces predominantly show blue band A luminescence. During growth the carbon atoms on these surfaces or at steps on these faces can

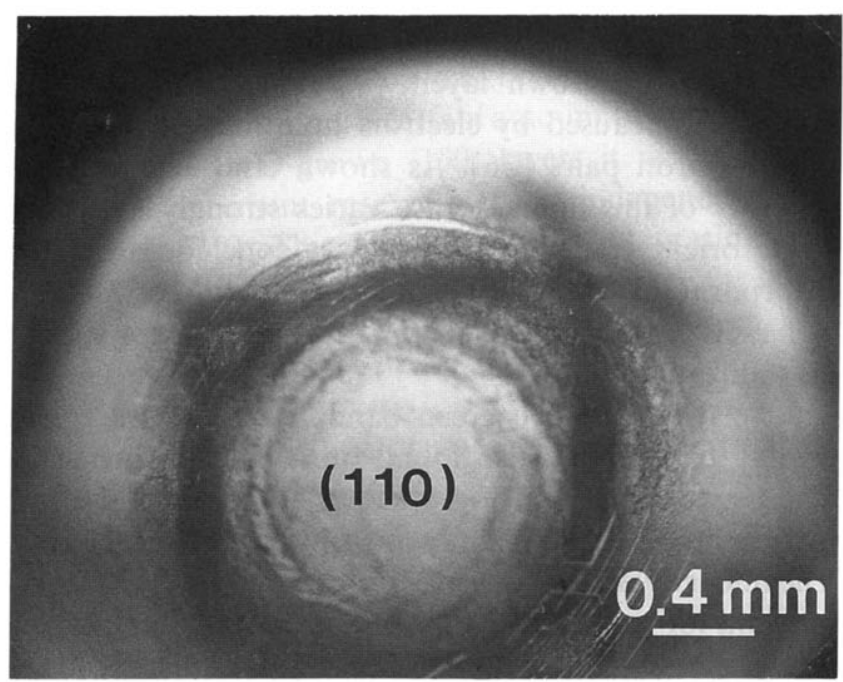

Fig. 10. CL topograph of the hemispheric single-crystal diamond substrate after deposition of a diamond layer by the flame technique $\left(S_{\text {ac }}=3.9 \%, T=1155^{\circ} \mathrm{C}\right)$. The bright surfaces correspond to $575 \mathrm{~nm}$ $\mathrm{N}-\mathrm{V}$ luminescence, the darker areas to blue band $\mathrm{A}$ luminescence. form dimers [31]. The remaining surface, in particular the $\{110\}$ faces and the $\langle 110\rangle$ zones between $\{111\}$ and $\{110\}$, is dominated by the $575 \mathrm{~nm}$ system. Here the surface atoms cannot dimerize [31]. This observation demonstrates beautifully that the formation of point defects is closely related to the interfacial processes, e.g. surface reconstruction, during diamond growth.

\section{Conclusions}

The deposition of diamond from the gas phase is determined by a complicated sequence of surface reactions in which the crystallographic orientation of the growing face plays a decisive role. The crystal forms $\{111\}$ and $\{100\}$ are "flat" faces, the latter of which is reconstructed at an atomic scale. Here growth proceeds via a layer mechanism involving steps emitted from "foreign" diamond particles or from lattice defects emerging at the surfaces. The slightly curved $\{113\}$ faces show a behaviour which is intermediate between "flat" and "rough". Faces of other orientations are "rough" and are stabilized by the development of microfacets. It is clear that in understanding CVD diamond growth one should be aware of the many-sidedness and subtlety of the surface phenomena encountered during this study. Therefore a relevant modelling of diamond crystallization remains a challenge for theorists.

\section{Acknowledgments}

The authors are grateful to the diamond workers at Drukker International B.V. for the careful polishing of the diamond substrates. Further, they acknowledge the Netherlands Technology Foundation (STW) for partial financial support.

\section{References}

1 P. Bennema, in R. G. Chambers, J. E. Enderby, A. Keller, A. R. Lang and J. W. Steeds (eds.), Sir Charles Frank, An Eightieth Birthday Tribute, Adam Hilger, Bristol, 1991, p. 46.

2 P. Hartman and W. G. Perdok, Acta Crystallogr., 8 (1955) 49, 521,525 .

3 J. P. van der Eerden, P. Bennema and T. A. Cherepanova, Prog. Cryst. Growth Charact., I (1978) 219.

4 P. Hartman, Z. Kristallogr., 121 (1965) 78.

5 L. J. Giling and W. J. P. van Enckevort, Surf. Sci., 161 (1985) 567.

6 W. J. P. van Enckevort, J. Hard Mater., 1 (1990) 247.

7 W. J. P. van Enckevort, G. Janssen, W. Vollenberg, M. Chermin, L. J. Giling and M. Seal, Surf. Coat. Technol., 47 (1991) 39.

8 W. J. P. van Enckevort, G. Janssen and L. J. Giling, J. Cryst. Growth, 113 (1991) 295.

9 G. Janssen, W. J. P. van Enckevort, W. Vollenberg and L. J. Giling, Diamond Relat. Mater., I (1992) 789. 
10 X. H. Wang, G.-H. M. Ma, W. Zhu, J. T. Glass, L. Bergman, K. F. Turner and R. J. Nemanich, Diamond Relat. Mater., I (1992) 828.

11 G. Janssen, W. J. P. van Enckevort, J. J. D. Schaminée, W. Vollenberg, L. J. Giling and M. Seal, J. Cryst. Growth, 104 (1990) 752.

12 J. J. Schermer, J. Hoogenkamp, G. C. J. Otter, G. Janssen, W. J. P. van Enckevort and L. J. Giling, Diamond Relat. Mater., 2 (1993) 1149.

13 W. J. P. van Enckevort, Prog. Cryst. Growth Charact., 9 (1984) 1.

14 W. J. P. van Enckevort, J. Cryst. Growth, 119 (1992) 177.

15 D. J. Marshall, Cathodoluminescence of Geological Materials, Unwin Hyman, London 1988.

16 G. Janssen, J. J. Schermer, W. J. P. van Enckevort and L. J. Giling, J. Cryst. Growth, 125 (1992) 42.

17 S. Szuba, W. J. P. van Enckevort and H. van Kempen, unpublished results.

18 Z. W. Hu, S. S. Jiang, P. Q. Huang, S. H. Li, Z. M. Zhang, C. Z. Ge, X. N. Zhao and D. Feng, J. Phys.: Condens. Matter, 4 (1992) 3753.

19 W. J. P. van Enckevort, G. Janssen, W. Vollenberg and L. J. Giling, submitted to J. Cryst. Growth.

20 P. Bennema and G. H. Gilmer, in P. Hartman (ed.), Crystal Growth: An Introduction, North-Holland, Amsterdam, 1973, p. 262.

21 H. Rau and F. Picht, J. Mater. Res., 7 (1992) 934.

22 K. Okada, S. Komatsu, T. 1shigaki, S. Matsumoto and Y. Moriyoshi, J. Appl. Phys., 71 (1992) 4920.

23 H. G. Busmann, W. Zimmerman-Edling, H. Sprang, H.-J. Güntherodt and I. V. Hertel, Diamond Relat. Mater., I (1992) 979.
24 K. Okada, S. Komatsu and Y. Moriyoshi, J. Chem. Vap. Depos., I (1992).

25 M. Kamo, H. Yurimoto and Y. Sato, Appl. Surf. Sci., 33-34 (1988) 553.

26 J. P. van der Eerden and H. Müller-Krumbhaar, Electrochim. Acta, 31 (1986) 1007.

27 T. Tsuno, T. Imai, Y. Nishibayashi, K. Hamada and N. Fujimori, Jpn. J. Appl. Phys., 30 (1991) 1063.

28 A. J. Hoeven, J. M. Lenssinck, D. Dijkkamp, E. J. van Loenen and J. Dieleman, Phys. Rev. Lett., 63 (1989) 1830.

29 D. J. Chadi, Phys. Rev. Lett., 59 (1987) 169l.

30 W. J. P. van Enckevort, P. Bennema and W. H. van der Linden, Z. Phys. Chem. N.F., 124 (1981) 171.

31 J. G. E. Gardeniers, W. E. J. R. Maas, R. Z. C. van Meerten and L. J. Giling, J. Cryst. Growth, 96 (1989) 832.

32 J. H. Wilson, P. D. Scott, J. B. Pethica and J. Knall, J. Phys.: Condens. Matter, 3 (1991) S133.

33 L. F. Sutcu, M. S. Thompson, C. J. Chu, R. H. Hauge, J. L. Margrave and M. P. D'Evelyn, Appl. Phys. Lett., 60 (1992) 1685.

34 R. Janssen van Rosmalen, W. H. van der Linden, E. Dobbinga and D. Visser, Kristall und Techniek, 13 (1978) 17.

35 P. Hartman, Neues Jahrb. Mineral. Monatsh., 4 (1959) 73.

36 H. Kawarada, Y. Yokota, Y. Mori, H. Nishimura and A. Hiraki, J. Appl. Phys., 67 (1990) 983.

37 A. T. Collins and S. C. Lawson, J. Phys.: Condens. Matter, I (I989) 4029.

38 P. J. Dean, Phys. Rev., 139 (1965) A588. 